\title{
Agrupamento de curvas de progresso de requeima, em tomateiro originado de cruzamento interespecífico
}

\author{
Cibelle Vilela Andrade Fiorini(1), Derly José Henriques da Silva(2), Fabyano Fonseca e Silva(3), \\ Eduardo Seiti Gomide Mizubuti(4), Daniel Pedrosa Alves( ${ }^{(2)}$ e Tiago de Sá Cardoso(2)
}

\begin{abstract}
(1)Universidade Federal Rural do Rio de Janeiro, Instituto de Agronomia, Departamento de Fitotecnia, BR 465, Km 7, CEP 23851-970 Seropédica, RJ. E-mail: cibellefiorini@ufrrj.br (2)Universidade Federal de Viçosa (UFV), Departamento de Fitotecnia, Avenida P.H. Rolfs, s/no, CEP 36570-000 Viçosa, MG. E-mail: derly@ufv.br, pedrosavrb@yahoo.com.br, thiagosacardoso@gmail.com (3)UFV, Departamento de Informática. E-mail: fabyano@dpi.ufv.br (4)UFV, Departamento de Fitopatologia. E-mail: mizubuti@ufv.br
\end{abstract}

Resumo - O objetivo deste trabalho foi estimar curvas de progresso de requeima, em genótipos de tomateiro, e identificar grupos de genótipos resistentes à doença. Foram avaliados 25 híbridos de tomateiro, originados de cruzamentos entre quatro variedades comerciais, um acesso do Banco de Germoplasma de Hortaliças (BGH), da Universidade Federal de Viçosa (UFV), e cinco linhagens $\mathrm{F}_{8}$ (Solanum lycopersicum x Solanum habrochaites), estas últimas selecionadas como fonte de resistência à requeima. As plantas foram inoculadas com uma mistura de esporângios de Phytophthora infestans e, em seguida, foram realizadas seis avaliações quanto à severidade de requeima, a intervalos de três dias. Ajustou-se o modelo exponencial aos dados de percentagem de severidade de requeima, e as estimativas obtidas quanto à incidência inicial da doença $\left(\mathrm{y}_{\mathrm{o}}\right) \mathrm{e}$ taxa de progresso da doença $(r)$ foram submetidas à análise de variância multivariada (Manova). As médias dessas estimativas, para cada genótipo, foram submetidas à análise de agrupamento. Observou-se um número ótimo de oito grupos distintos, o que possibilitou identificar genótipos resistentes e suscetíveis. Os híbridos experimentais Ikram x 73 A, Nemo-Netta x 133 A, Ikram x 163 A e Nemo-Netta x 163 A apresentaram a menor taxa de progresso de requeima e, portanto, maior resistência à doença.

Termos para indexação: Phytophtora infestans, Solanum, análise de grupo, melhoramento genético, resistência genética.

\section{Clustering of progress curves of late blight for tomato genotypes from interspecific crosses}

\begin{abstract}
The objective of this work was to estimate the progress curves of late blight in tomato genotypes, and to identify tomato genotype groups resistant to this disease. Twenty-five hybrid tomatoes, originated from crosses between four fresh market cultivars, one access from the Banco de Germoplasma de Hortaliças (BGH) of Universidade Federal de Viçosa (UFV), and five $\mathrm{F}_{8}$ lines (Solanum lycopersicum x Solanum habrochaites), selected as late blight resistance source, were evaluated. Plants were inoculated with a sporangium mixture of Phytophthora infestans, and then, six evaluations for late blight severity were carried out every three days. The exponential model was fitted to the data of late blight severity percentage, and the obtained parameter estimates - initial incidence of disease $\left(\mathrm{y}_{\mathrm{o}}\right)$ and progress rate of disease $(\mathrm{r})$ - were submitted to the multivariate analysis of variance (Manova). The means of these estimates, obtained for each genotype, were submitted to the cluster analysis. An optimal number of eight distinct groups was observed, which made it possible to identify the genotypes belonging both to the resistant and susceptible groups. The experimental hybrids Ikram x $73 \mathrm{~A}$, Nemo-Netta x 133 A, Ikram x 163 A and Nemo-Netta x 163 A showed the smaller rate of disease progress and, therefore, higher late blight resistance.
\end{abstract}

Index terms: Phytophtora infestans, Solanum, cluster analysis, plant breeding, genetic resistance.

\section{Introdução}

$\mathrm{Na}$ cultura do tomateiro, a mela ou requeima, causada por Phytophthora infenstans (Mont.) De Bary, é considerada uma doença destrutiva que pode, sob condições climáticas favoráveis, causar severas epidemias no campo e perda total da produção (Mizubuti, 2001, 2005; Vale et al., 2007).
No Brasil, o manejo da requeima na cultura do tomateiro é feito exclusivamente pelo princípio de proteção do hospedeiro, por meio da aplicação de fungicidas de caráter preventivo e curativo, num total de 15 a 25 aplicações por safra (Costa et al., 2007). Segundo estimativas de economistas do Centro Internacional de La Papa (CIP), mundialmente são gastos cerca de um bilhão de dólares por ano no controle dessa doença (Mizubuti, 2005; Mizubuti \& Fry, 2006).

Pesq. agropec. bras., Brasília, v.45, n.10, p.1095-1101, out. 2010 
As variedades e híbridos de tomateiro cultivados no Brasil são suscetíveis à requeima (Mizubuti, 2005; Reis et al., 2006; Costa et al., 2007; Vale et al., 2007). Não há genótipos com boas características agronômicas e comerciais que apresentem nível satisfatório de resistência à requeima (Mizubuti, 2005), e a resistência a diversos patógenos é comumente encontrada em espécies silvestres, como Solanum habrochaites f. glabratum (Laterrot, 2000; Abreu et al. 2008).

Entre as metodologias disponíveis para identificação de genótipos resistentes a patógenos, destaca-se a análise de curvas de progresso da doença, que expressa a proporção da doença em função do tempo, e representa todas as interações que ocorrem entre patógeno e hospedeiro, sob a influência do ambiente (Jesus Júnior et al., 2004). Essas curvas podem ser descritas por meio de modelos específicos, cujos parâmetros apresentam interpretação biológica, tais como incidência inicial e taxa de progresso, cujos valores são diretamente proporcionais à quantidade de doença (Campbell \& Madden, 1990).

Geralmente, em estudos de curvas de progresso de doença, quando existem vários tratamentos, é usual comparar a forma da curva, a fim de identificar quais delas proporcionam menor intensidade de doença. Porém, na presença de um elevado número de tratamentos, alguns métodos tradicionais de comparação duas a duas, como os descritos por Campbell \& Maden (1990), tornam-se inviáveis. Dessa forma, a análise de agrupamento(Khattree \& Naik, 2000) representa uma solução viável para esse problema, uma vez que possibilita agrupar tratamentos semelhantes em relação às curvas, ou seja, tratamentos que apresentam vetores similares das estimativas dos parâmetros do modelo de progresso da doença.

O objetivo deste trabalho foi estimar curvas de progresso de requeima, em genótipos de tomateiro, e identificar, mediante análise de agrupamento para as médias das estimativas dos parâmetros das curvas, grupos de genótipos resistentes à doença.

\section{Material e Métodos}

O experimento foi conduzido em campo, no período de 30 de maio a 29 de julho de 2007, na horta de pesquisa da Universidade Federal de Viçosa (UFV) - Horta Velha -, em Viçosa, MG (2045'14"S; 4252'53"W; 648,74 m de altitude). Segundo a classificação de Köppen, o clima regional é do tipo Cwa, com umidade relativa média anual do ar de $80 \%$, temperaturas médias máxima e mínima anual registradas de 26,4 e $14,8^{\circ} \mathrm{C}$, respectivamente, e precipitação média anual de $1.221,4 \mathrm{~mm}$. Entre os dias 11 de julho e 29 de julho, período entre a inoculação e a última avaliação de severidade da requeima, foram observadas no Município de Viçosa, MG temperaturas médias diárias, máxima e mínima de 25,5 e $12,8^{\circ} \mathrm{C}$, respectivamente, umidade relativa média de $77 \%$ e precipitação de $6,1 \mathrm{~mm}$.

Foram avaliados 25 cinco híbridos experimentais, provenientes de cruzamentos entre quatro variedades comerciais de tomateiro, para consumo in natura, o acesso BGH 1497, e mais cinco linhagens $\mathrm{F}_{8}$ (Solanum lycopersicum L. x Solanum habrochaites Knapp \& Spooner), selecionadas como fonte de resistência à requeima (Tabela 1). Além dos híbridos experimentais, foram incluídos nos tratamentos a cultivar Santa Clara e o acesso BGH 6902, utilizados como testemunhas suscetível e resistente à requeima, respectivamente.

Na obtenção dos híbridos experimentais, as variedades comerciais Alambra, Fanny, Ikram e Nemo-Netta, além do acesso BGH 1497, foram utilizadas como genitores femininos $\mathrm{e}$ as cinco linhagens $\mathrm{F}_{8}$ como genitores masculinos. As linhagens $F_{8}$, identificadas como $64 \mathrm{~B}, 73 \mathrm{~A}, 127 \mathrm{~F}, 133 \mathrm{~A}$ e $163 \mathrm{~A}$, foram originadas de cruzamento interespecífico entre a cultivar Santa Clara (S. lycopersicum) e o acesso BGH 6902 (S. habrochaites), conforme Abreu et al. (2008), e, nas gerações $\mathrm{F}_{5}$ e $\mathrm{F}_{8}$, selecionadas como fonte de resistência à requeima (Abreu, 2005; Fiorini et al., 2010). O acesso BGH 1497 destacouse, entre 72 genótipos de tomateiro avaliados, por possuir bom nível de resistência à requeima (Fiorini et al., 2010).

A semeadura dos genótipos foi feita em bandejas de isopor de 128 células, contendo substrato comercial para hortaliças, com cinco sementes por célula. Após a germinação, procedeu-se ao desbaste. Ao apresentar quatro folhas definitivas, as plântulas foram transferidas para copos de plásticos descartáveis com substrato comercial para hortaliças. Durante a fase de produção de mudas, desde a semeadura até o transplantio para o campo, os genótipos foram mantidos em casa de vegetação, e as bandejas irrigadas com regador, três vezes ao dia, às 9, 12 e 16h30min. O transplantio no campo foi feito no dia 30 de maio de 2007, em espaçamento de 1,00x0,50 $\mathrm{m}$. Utilizou-se o delineamento experimental utilizado de blocos ao acaso, com duas repetições e cinco plantas por parcela, com área útil de $2,5 \mathrm{~m}^{2}$ por parcela. Foram utilizadas plantas da cultivar Santa Clara como bordadura ao redor de todo o experimento. As plantas foram 
conduzidas com uma haste, em sistema de tutoramento com fitilho na vertical.

No dia 11 de julho de 2007, as plantas foram inoculadas com mistura de esporângios de cinco isolados de Phytophthora infestans patogênicos ao tomateiro, na concentração de $10^{3}$ esporângios $\mathrm{mL}^{-1}$, coletados em campos de produção nos municípios mineiros de Cajuri, Coimbra, Ervália e Viçosa (Zona Rural e Horta de Pesquisa da UFV, Horta Velha). O inóculo foi multiplicado no Laboratório de Epidemiologia do Departamento de Fitopatologia da UFV. Os folíolos infectados foram transferidos para bandejas de plástico, identificadas, desinfestadas em álcool a 70\% e forradas com papel toalha umedecido em água destilada. Cada bandeja foi coberta com plástico e mantida em câmara de crescimento a $18^{\circ} \mathrm{C}$, por 24 horas, para induzir a esporulação do patógeno. As partes infectadas dos folíolos em que se observou esporulação do patógeno foram retiradas, colocadas em um Becker com água destilada, e levadas a agitador por $5 \mathrm{~min}$, para liberação dos esporângios. A suspensão de esporângios foi homogeneizada, e ajustou-se a concentração do hemacitômetro para $10^{3}$ esporângios $\mathrm{mL}^{-1}$. Nas inoculações, foram utilizadas suspensões de volumes iguais para cada isolado, que constituíram a mistura de esporângios. Após a contagem do número de esporângios, a suspensão foi levada à geladeira por uma hora, para liberação de zoósporos. A inoculação foi feita às $21 \mathrm{~h}$, com o auxílio de um pulverizador costal manual, com a pulverização de $20 \mathrm{~mL}$ da suspensão de esporângios em cada planta. $\mathrm{O}$ tempo decorrido entre o preparo da suspensão de esporângios e a inoculação não excedeu duas horas, para que os zoósporos não se tornassem inviáveis (Abreu, 2005; Fiorini, 2008).

Com o intuito de garantir alta umidade ao ambiente e a infecção pelo patógeno, 12 horas após a inoculação, as plantas passaram a ser irrigadas por aspersão três vezes ao dia, às 10,13 e $17 \mathrm{~h}$, por $20 \mathrm{~min}$, entre os dias $12 \mathrm{e}$ 29/7/2007.

Três dias após a inoculação, foram feitas as avaliações de severidade da requeima. Foram realizadas seis avaliações, em intervalos de três dias. Para a realização dessas avaliações, os avaliadores foram treinados para usar o programa Severity PRO (Nutter, 1997), com o objetivo de corrigir distorções inerentes à estimativa visual de severidade da doença. No campo, os avaliadores treinados estimaram, visualmente, sem auxílio de escala diagramática, a severidade da doença. Nas avaliações, foram consideradas todas as folhas de uma planta e todas as cinco plantas das parcelas experimentais. A nota final de cada planta foi dada pela média dos valores de severidade de suas folhas.

Durante a condução do experimento, as plantas foram irrigadas e adubadas regularmente, e receberam os tratos normais da cultura, como desbrotas, amarrios e tratamentos fitossanitários, de acordo com Alvarenga (2004). A irrigação, até a data anterior à inoculação, foi realizada três vezes por semana, por sulcos. Foram feitos os tratamentos fitossanitários recomendados para a cultura, segundo Alvarenga (2004). Entretanto, devido ao prazo de carência dos fungicidas e às avaliações para severidade de requeima, esses tratamentos foram interrompidos dez dias antes da inoculação e durante as avaliações do experimento.

$\mathrm{O}$ modelo exponencial $\mathrm{y}_{\mathrm{i}}=\mathrm{y}_{\mathrm{o}} \exp \left(\mathrm{rt}_{\mathrm{i}}\right)+\varepsilon_{\mathrm{i}}$, para $\mathrm{i}=1,2, \ldots, 6$, foi ajustado aos dados de percentagem de severidade (y) da requeima, com o intuito de descrever seu comportamento em função do tempo $(\mathrm{t})$, sendo $\mathrm{y}_{\mathrm{o}}$ a quantidade de inóculo inicial, $r$ a taxa de progresso da doença, e $\varepsilon_{\mathrm{i}}$ o termo de erro aleatório, $\varepsilon_{\mathrm{i}} \sim \mathrm{N}\left(0, \sigma^{2}\right)$, tendo sido consideradas as seis avaliações realizadas. Tal ajuste foi realizado para cada repetição de cada genótipo, mediante o Proc Model (SAS Institute, 1996), o que possibilitou a obtenção de um arquivo com as estimativas geradas por $\mathrm{y}_{\mathrm{o}}$ e r. Posteriormente, foi feita a análise de variância multivariada, utilizando-se o Proc GLM (SAS Institute, 1996), para avaliar a influência dos genótipos simultaneamente sobre as estimativas dos parâmetros $\mathrm{y}_{\mathrm{o}}$ e r (Khattree \& Naik, 2000).

Para avaliar a normalidade dos resíduos requeridos pela Manova, utilizou-se o teste de Shapiro-Wilk generalizado para distribuições multivariadas (Royston, 1982), que foi implementado pela função mshapiro.test do pacote mvnormtest do programa R (R Development Core Team, 2010). Uma vez verificada a condição de normalidade residual e, constatada a influência dos genótipos sobre as estimativas de $y_{0}$ e r, extraíram-se as médias ajustadas por quadrados mínimos (LSMeans) e foram consideradas duas repetições. Tais médias foram submetidas à análise de agrupamento, no Proc Cluster (SAS Institute, 1996), pelo método centroide.

Nessa última análise, foram obtidos os valores dos desvios-padrão da raiz do quadrado médio (RMSSTD), em relação ao número de agrupamentos, e foi gerado um gráfico com a disponibilização ou a identificação do número ótimo de agrupamentos, em relação à máxima curvatura, de acordo com método gráfico apresentado 
por Cecon et al. (2008). Para complementar a análise, foi utilizado o Proc Tree (SAS Institute, 1996), para visualizar o dendrograma e verificar quais genótipos pertenciam aos diferentes grupos obtidos pela discriminação estatística, em relação aos vetores dos parâmetros $y_{o}$ e r. Para cada grupo obtido, foi gerada uma curva média de progresso da requeima.

\section{Resultados e Discussão}

Os ajustes do modelo exponencial aos dados de severidade para cada repetição de cada genótipo proporcionaram coeficientes de determinação $\left(\mathrm{R}^{2}\right)$ em torno de $99 \%$, indicativos de que o modelo utilizado é adequado para descrever curvas de progresso da requeima para todos os genótipos avaliados (Tabela 1). Em relação à qualidade desse modelo, Souza (2006) estudou a mancha-parda do pessegueiro e observou que o $\mathrm{R}^{2}$ variou entre 0,85 e 0,94 , para os tratamentos considerados. Quanto à aplicabilidade do modelo em questão, Dannon \& Wydra (2004) estudaram o progresso de Ralstonia solanacearum, em genótipos de tomateiro, e relataram que foi possível detectar genótipos tolerantes de acordo com as estimativas dos parâmetros $\mathrm{y}_{\mathrm{o}} \mathrm{e} \mathrm{r}$.

Os resultados das estimativas dos parâmetros do ajuste do modelo exponencial, utilizadas como variáveis dependentes na análise de variância multivariada (Manova), mostraram efeito significativo dos genótipos de tomateiro sobre o vetor de parâmetros $\mathrm{y}_{\mathrm{o}}$ e r, a $1 \%$ de probabilidade (Tabela 1). O teste de Shapiro-Wilk, generalizado para distribuições multivariadas, mostrou que os resíduos da Manova distribuíam-se como uma normal multivariada $(\mathrm{p}=0,0756)$. Caso o teste apresentasse $\mathrm{p}<0,05$, a condição de normalidade não seria aceita.

Tabela 1. Genótipos, testemunha resistente e suscetivel, utilizados nas avaliações para resistência à requeima e ajuste do modelo exponencial aos dados de severidade de requeima para 25 híbridos experimentais.

\begin{tabular}{|c|c|c|c|c|c|}
\hline Genótipos & Cruzamentos $^{(1)}$ & Media $\mathrm{y}_{\mathrm{o}}$ & $\mathrm{DP} \mathrm{y}_{\mathrm{o}}$ & Media r & $\mathrm{DP} \mathrm{r}$ \\
\hline 1 & Alambra x $127 \mathrm{~F}$ & 0,424865 & 0,373119 & 0,238465 & 0,013131 \\
\hline 2 & Alambra x 64 B & 0,350690 & 0,210732 & 0,259850 & 0,072733 \\
\hline 3 & Alambra x 3 A & 0,635430 & 0,454161 & 0,224065 & 0,004900 \\
\hline 4 & Alambra x 163 A & 0,324970 & 0,189773 & 0,287115 & 0,060804 \\
\hline 5 & Alambra x $133 \mathrm{~A}$ & 0,691455 & 0,163589 & 0,284925 & 0,017161 \\
\hline 6 & BGH 1497 x $127 \mathrm{~F}$ & 0,780710 & 0,750636 & 0,190495 & 0,029239 \\
\hline 7 & BGH 1497 x 64 B & 1,068995 & 0,734168 & 0,185600 & 0,021298 \\
\hline 8 & BGH 1497 x 73 A & 0,957725 & 0,238571 & 0,226340 & 0,008938 \\
\hline 9 & BGH 1497 x 163 A & 0,239065 & 0,079599 & 0,314610 & 0,002376 \\
\hline 10 & BGH 1497 x 133 A & 0,550555 & 0,487020 & 0,274790 & 0,056328 \\
\hline 11 & Fanny x $127 \mathrm{~F}$ & 0,543345 & 0,537267 & 0,283915 & 0,049391 \\
\hline 12 & Fanny x 64 B & 0,374990 & 0,370849 & 0,311430 & 0,078432 \\
\hline 13 & Fanny x 73 A & 0,182160 & 0,052397 & 0,346260 & 0,016263 \\
\hline 14 & Fanny x 163 A & 0,231365 & 0,004632 & 0,299390 & 0,029020 \\
\hline 15 & Fanny x 133 A & 0,286095 & 0,007715 & 0,306965 & 0,004929 \\
\hline 16 & Ikram x $127 \mathrm{~F}$ & 0,152490 & 0,037293 & 0,285155 & 0,027344 \\
\hline 17 & Ikram x 64 B & 0,448545 & 0,440181 & 0,254450 & 0,049356 \\
\hline 18 & Ikram x 73A & 0,105560 & 0,011257 & 0,330890 & 0,004384 \\
\hline 19 & Ikram x $163 \mathrm{~A}$ & 0,093840 & - & 0,304830 & - \\
\hline 20 & Ikram x 133 A & 0,823295 & 0,469356 & 0,225155 & 0,039817 \\
\hline$\overline{21}$ & Nemo-Netta x $127 \mathrm{~F}$ & 0,401325 & 0,065287 & 0,251830 & 0,033474 \\
\hline 22 & Nemo-Netta x 64 B & 0,258690 & 0,110606 & 0,290150 & 0,048960 \\
\hline 23 & Nemo-Netta x 73 A & 0,206415 & 0,085751 & 0,317860 & 0,015358 \\
\hline 24 & Nemo-Netta x 163 A & 0,077720 & - & 0,377280 & - \\
\hline 25 & Nemo-Netta x 133 A & 0,105825 & 0,001393 & 0,328265 & 0,074409 \\
\hline 26 & Santa Clara & \multicolumn{4}{|c|}{ Testemunha susceptível (Abreu et al., 2008), cultivar comercial, Isla Sementes, Porto Alegre, RS. } \\
\hline 27 & BGH $6902^{(2)}$ & \multicolumn{4}{|c|}{ Testemunha resistente (Abreu et al., 2008; Fiorini et al., 2010), S. habrochaites Knapp \& Spooner. } \\
\hline \multicolumn{6}{|c|}{$\begin{array}{l}\text { (1) } 25 \text { cruzamentos entre cultivares comerciais de tomateiro para consumo in natura, um acesso do BGH-UFV (1497) e cinco linhagens F8 de Solanum } \\
\text { lycopersicum x Solanum habrochaites, identificadas como: } 127 \text { F, } 64 \text { B, } 73 \text { A, } 163 \text { A e } 133 \text { A, selecionadas como fonte de resistência à requeima, conforme } \\
\text { Fiorini et al. (2010). Cultivares comerciais: Alambra, Clause Vegetable Seeds, Campinas, SP; Fanny, Seminis, Campinas- SP; Ikram, Syngenta, Santo Amaro- SP; } \\
\text { Nemo-Netta, Agrocinco, Monte Mor - SP. Acesso BGH } 1497 \text { (Solanum lycopersicum L.): Banco de Germoplasma de Hortaliças da UFV, coletado em } 1967 \\
\text { na extinta Cooperativa Agrícola de Cotia (CAC), no Estado de São Paulo (www.ufv.br/bgh). }{ }^{(2)} \text { Acesso BGH } 6902: \text { Banco de Germoplasma de Hortaliças da } \\
\text { UFV. DP, desvio-padrão; yo, quantidade de inóculo inicial; r, taxa de progresso da requeima; (-), parcela perdida. }\end{array}$} \\
\hline
\end{tabular}

Pesq. agropec. bras., Brasília, v.45, n.10, p.1095-1101, out. 2010 
O número ótimo de grupos, determinado de acordo com o comportamento da estatística RMSSTD, em razão do número de agupamentos, foi oito (Figura 1). Observase que, a partir de oito grupos, os valores RMSSTD continuam decrescendo, porém muito lentamente, e indica que a formação de novos agrupamentos já não reduz efetivamente o RMSSTD. De acordo com Cecon et al. (2008), que usaram essa mesma metodologia para agrupar curvas de produção de cafeeiros, a utilização da estatística RMSSTD evita a escolha subjetiva do número de agrupamentos, o que confere maior confiabilidade àqueles obtidos.

A cultivar Santa Clara e o acesso BGH 6902, considerados respectivamente como testemunhas suscetível e resistentes à requeima, ficaram alocados em grupos distintos (Figura 2), o que evidencia a diferença entre as curvas de progresso da doença desses dois genótipos (Figura 3).

A cultivar Santa Clara apresentou os maiores valores para a incidência inicial $\left(\mathrm{y}_{\mathrm{o}}\right)$ e taxa de progresso de requeima (r), e foi alocada no grupo VIII (Figura 2). $\mathrm{O}$ acesso BGH 6902, pertencente ao grupo VII, apresentou comportamento diferenciado em relação aos

Tabela 2. Estatísticas utilizadas para verificação do efeito dos genótipos sobre o vetor de parâmetros $\mathrm{y}_{\mathrm{o}}$ e $\mathrm{r}$ na análise de variância multivariada (Manova).

\begin{tabular}{lcccc}
\hline Estatística & Valor da estatística & Valor de F & GL & P \\
\hline Wilks' lambda & 0,03640271 & 3,91 & 52 & $<0,001$ \\
Pillai's trace & 1,60541669 & 3,91 & 52 & $<0,001$ \\
Hotelling-lawley & 8,83939471 & 3,94 & 52 & $<0,001$ \\
Roy's root & 5,79905776 & 5,58 & 26 & $<0,001$ \\
\hline
\end{tabular}

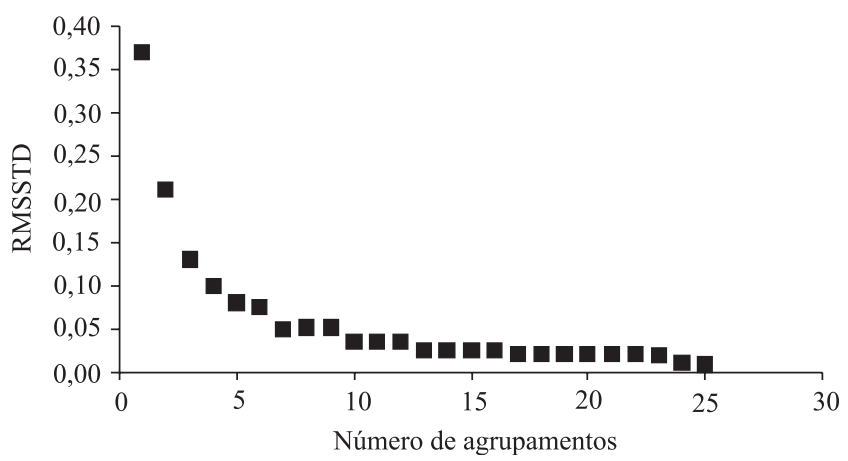

Figura 1. Comportamento da estatística desvio-padrão da raiz do quadrado médio (RMSSTD), em razão do número de agrupamentos. demais genótipos, pois até o décimo segundo dia após a inoculação foi observado um progresso de requeima muito inferior ao da cultivar Santa Clara (Figura 3). Após esse período, o progresso da requeima elevou-se

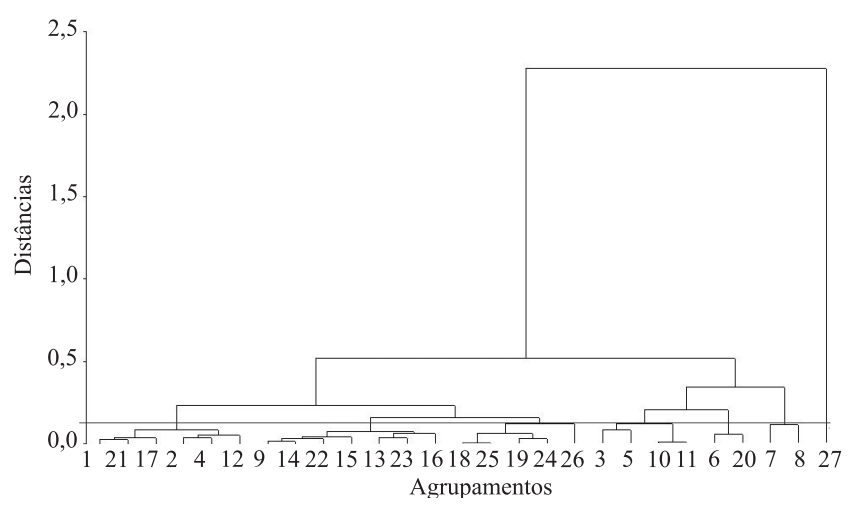

Figura 2. Dendrograma proveniente da análise de agrupamento para classificação dos genótipos, considerando as estimativas dos parâmetros $\mathrm{y}_{\mathrm{o}} \mathrm{e} \mathrm{r}$ das curvas de progresso da requeima do tomateiro. Grupo I, genótipos 18, 25, 19, 24. Grupo II, genótipos 3, 5, 10, 11. Grupo III, genótipos 9, 14, 22, 15, 13, 23, 16. Grupo IV, genótipos 1, 21, 17, 2, Alambra x 64 B; 4, 12. Grupo V, genótipos 6, 20. Grupo VI, genótipos 7, 8. Grupo VII, genótipo 26, e Grupo VIII, genótipo 27.

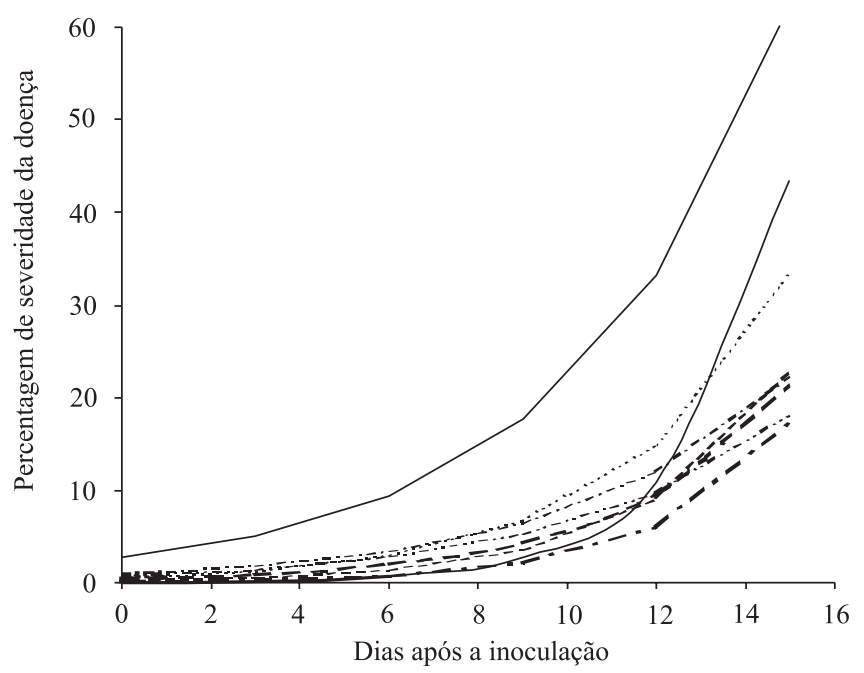

$$
\begin{aligned}
& \text { - - - Grupo I: } \mathrm{y}=0,0857175 \operatorname{EXP}(0,3543051 \mathrm{x}) \quad \mathrm{R}^{2}=0,93 \\
& \text { - . - . - . Grupo II: } y=0,6052125 \operatorname{EXP}(0,2669175 x) \quad R^{2}=0,96 \\
& \text { - - - - Grupo III: } y=0,2223542 \operatorname{EXP}(0,3086157 x) \quad R^{2}=0,94 \\
& \text { - - Grupo IV: } \mathrm{y}=0,3876116 \operatorname{EXP}(0,2671783 \mathrm{x}) \quad \mathrm{R}^{2}=0,93 \\
& \text { - - - - Grupo V: } y=0,8019222 \operatorname{EXP}(0,2078259 x) \quad R^{2}=0,93 \\
& \text { - - - - Grupo VI: } \mathrm{y}=1,0131751 \operatorname{EXP}(0,2059822 x) \quad \mathrm{R}^{2}=0,97 \\
& \text { Grupo VII: } y=0,0412612 \operatorname{EXP}(0,4639222 x) \quad R^{2}=0,97 \\
& \text { Grupo VIII: } y=2,6813812 \operatorname{EXP}(0,2096800 x) R^{2}=0,96
\end{aligned}
$$

Figura 3. Curvas de progresso da requeima, obtidas em oito grupos de tomateiro com diferentes genótipos. 
abruptamente, superando inclusive as proporções finais de doença obtidas para os grupos I, II, III, IV, V e VI. Foi observada, para a curva de progresso de requeima no acesso BGH 6902, uma proporção final de doença em torno de 69\% do valor observado para a cultivar Santa Clara, pertencente ao Grupo VIII. No entanto, esse acesso foi considerado como fonte de resistência à requeima em trabalhos de Abreu et al. (2008) e Fiorini et al. (2010).

Nas curvas de progresso de requeima, foi observada menor taxa de progresso da doença no Grupo I, sendo a proporção final de doença observada em cerca de $28 \%$, em relação ao valor obtido para a cultivar Santa Clara (Grupo VIII) (Figura 3). No presente trabalho, em razão de as condições climáticas e de o manejo aplicado à cultura ter sido o mesmo para todos os genótipos, pode-se inferir que a menor taxa de progresso de requeima, observada no Grupo I, foi ocasionada por diferença na resistência genética entre genótipos, e os híbridos experimentais Ikram x 73 A, NemoNetta x 133 A, Ikram x 163 A e Nemo-Netta x 163 A foram os que apresentaram maior resistência.

\section{Conclusão}

1. A análise de agrupamento das curvas de progresso da doença permitiu identificar oito grupos distintos quanto à resistência à requeima do tomateiro.

2. Os híbridos experimentais Ikram x 73 A, NemoNetta x 133 A, Ikram x 163 A, Nemo-Netta x 163 A possuem menor taxa de progresso de requeima e, consequentemente, maior resistência à doença.

\section{Agradecimentos}

À Coordenação Aperfeiçoamento de Pessoal de Nível Superior e ao Conselho Nacional de Desenvolvimento Científico e Tecnológico, pela bolsa de doutorado à primeira autora; à Fundação de Amparo à Pesquisa e Extensão do Estado de Minas Gerais; e à Universidade Federal de Viçosa, pelo auxílio financeiro e suporte na realização deste trabalho.

\section{Referências}

ABREU, F.B. Herança da resistência a Phytophthora infestans, de características de frutos e seleção de genótipos resistentes na geração $\mathbf{F}_{5}$ de cruzamento interespecífico em tomateiro. 2005. 95p. Tese (Doutorado) - Universidade Federal de Viçosa, Viçosa.
ABREU, F.B.; SILVA, D.J.H. da; CRUZ, C.D.; MIZUBUTI, E.S.G. Inheritance of resistance to Phytophthora infestans (Peronosporales, Pythiaceae) in a new source of resistance in tomato (Solanum sp. (formerly Lycopersicon sp.), Solanales, Solanaceae). Genetics and Molecular Biology, v.31, p.493-497, 2008.

ALVARENGA, M.A.R. (Ed.). Tomate: produção em campo, em casa-de-vegetação e em hidroponia. Lavras: UFLA, 2004. 393p.

CAMPBELL, C.L.; MADDEN, L.V. Introduction to plant disease epidemiology. New York: John Willey \& Sons, 1990.

CECON, P.R.; SILVA, F.F. e; FERREIRA, A.; FERRÃO, R.G.; CARNEIRO, A.P.S.; DETMANN, E.; FARIA, P.N.; MORAIS, T.S. da S. Análise de medidas repetidas na avaliação de clones de café 'Conilon'. Pesquisa Agropecuária Brasileira, v.43, p.1171-1176, 2008.

COSTA, H.; ZAMBOLIM, L.; VETURA, J.A. Doenças de hortaliças que se constituem em desafio para o controle. In: ZAMBOLIM, L.; LOPES, C.A.; PICANÇO, M.C.; COSTA, H. (Ed.). Manejo integrado de doenças e pragas: hortaliças. Viçosa: UFV, 2007. p.319-348.

DANNON, E.A.; WYDRA, K. Interaction between silicon amendment, bacterial wilt development and phenotype of Ralstonia solanacearum in tomato genotypes. Physiologycal and Molecular Plant Pathology, v.64, p.233-243, 2004.

FIORINI, C.V.A. Introgressão de genes de resistência à requeima de Solanum habrochaites em Solanum lycopersicum. 2008. 163p. Tese (Doutorado) - Universidade Federal de Viçosa, Viçosa.

FIORINI, C.V.A.; SILVA, D.J.H. da; MIZUBUTI, E.S.G.; BARROS, J. de S.; SILVA, L.J. da; MILAGRES, C.; ZAPAROLI, M.R. Caracterização de linhagens de tomateiro originadas de cruzamento interespecífico quanto à resistência à requeima. Horticultura Brasileira, v.28, p.197-202, 2010.

JESUS JÚNIOR, W.C. de; POZZA, E.A.; VALE, F.X.R. do; AGUILERA, G.M. Análise temporal de epidemias. In: VALE, F.X.R. do; JESUS JÚNIOR, W.C. de; ZAMBOLIM, L. (Ed.). Epidemiologia aplicada ao manejo de doenças de plantas. Belo Horizonte: Perfil, 2004. p.127-191.

KHATTREE, R.; NAIK, D.N. Multivariate data reduction and discrimination with SAS software. Cary: SAS Institute, 2000. $574 \mathrm{p}$.

LATERROT, H. Disease resistance in tomato: practical situation. Acta Physiologiae Plantarum, v.22, p.328-331, 2000.

MIZUBUTI, E.S.G. Custo da requeima. Cultivar Hortaliças e Frutas, v.32, p.23-26, 2005.

MIZUBUTI, E.S.G. Requeima ou mela da batata e do tomate. In: LUZ, E.D.M.N.; SANTOS, A.F. dos; MATSUOKA, K.; BEZERRA, J.L. (Ed.). Doenças causadas por Phytophthora no Brasil. Campinas: Rural, 2001. p.100-174.

MIZUBUTI, E.S.G.; FRY, W.E. Potato late blight. In: COOKE, B.M.; JONES, D.G.; KAYE, B. (Ed.). The epidemiology of plant diseases. Springer: Dordrecht, 2006. p.445-471.

NUTTER, J.R.F.W. Disease severity assessment training. In: FRANCL, L.J.; NEHER, D.A. (Ed.). Exercises in plant disease epidemiology. St. Paul: The American Phytopatological Society, 1997. p.1-7. 
R DEVELOPMENT CORE TEAM. R: a language and environment for statistical computing. Vienna: R Foundation for Statistical Computing, 2010. Available at: <http://www.R-project.org>. Accessed on: 11 Oct. 2010.

REIS, A.; RIBEIRO, F.H.S.; MIZUBUTI, E.S.G. Caracterização de isolados de Phytophthora infestans do Distrito Federal e de Goiás. Fitopatologia Brasileira, v.31, p.270-276, 2006.

ROYSTON, J.P. An extension of shapiro and Wilk's $W$ test for normality to large samples. Applied Statistics, v.31, p.115-124, 1982.
SAS INSTITUTE. SAS user's guide: statistics. Version 6.11. Cary: SAS Insitute, 1996. 842p.

SOUZA, D.C. de. Progresso temporal e padrão espacial de epidemias da podridão parda do pessegueiro. 2006. 64p. Tese (Doutorado) - Escola Superior de Agricultura Luiz de Queiroz, Piracicaba.

VALE, F.X.R.; JESUS JÚNIOR, W.C.; RODRIGUES, F.A.; COSTA, H.; SOUZA, C.A. Manejo de doenças fúngicas em tomateiro. In: SILVA, D.J.H.; VALE, F.X.R. (Ed.). Tomate: tecnologia de produção. Viçosa: UFV, 2007. p.159-197.

Recebido em 8 de julho de 2010 e aprovado em 29 de setembro de 2010 